\title{
Discussion of Effective Curriculum Creation by Comparing the Bilingual Acquisition Process
}

\author{
Jingru Yang \\ Gradual School of Harbin Normal University, Harbin, China \\ Xueyunerr@163.com
}

\begin{abstract}
Through the difference comparison of bilingual acquisition, it is found that bilingual learners are superior to single language learners in many aspects, such as cognitive ability, executive ability, ability to solve problems, multi task switching and management ability, filtering useless information, overcoming external interference and better social behavior. This paper aims to exploring the competence advantage of bilingual learners by comparing the process of bilingual acquisition in details between bilingual and monolingual learners from three aspects: input environment (including micro system and macro system), input approach (auditory, visual, multimodal sensory connection) and language processing (physiological basis, personality traits, self-image, and memory). And then put forward some suggestions for creating effective courses.
\end{abstract}

Keywords: bilingual acquisition; difference comparison; superiority; effective curriculum.

\section{双语习得差异性比较对有效课程创建的启发}

\author{
杨晶茹 \\ 哈尔滨师范大学研究生院, 哈尔滨, 中国
}

摘要: 经过双语习得差异性对比发现, 双语者在许多方面优于单语者, 包括认知能力、执行 能力、解决问题的能力、多重任务切换与管理能力、过滤无用信息、克服外在干扰、社会性 行为等。本文首先就双语习得过程进行对比, 从输入环境（微观系统、宏观系统）、输入途 径（听觉、视觉、多模态感官联结）、语言加工（生理基础、人格特质、自我形象、记忆） 三个方面探索双语者与单语学习者习得过程的差异以及双语者能力优势的原因, 然后为创建 有效课程提出若干建议。

关键词：双语习得；差异性比较；优势；有效课程

1. 双语习得过程对比

1.1 输入环境

1.1.1微观系统

根据布朗伦芬纳的生态系统理论, 早期儿童在微观系统中主要受到家庭的影响。成人语言对 儿童的影响主要是成人的语言习惯、语言支架详尽性等。虽然此阶段儿童模仿成人，但儿童 是选择性模仿, 选择性模仿的内容更多的是与自身经验相关的。也就是说, 儿童语言习得具 有自主选择性、经验偏好性。目前中国绝大多数家庭能够为儿童提供汉语环境, 少数家庭能 够提供原生态双语环境。从一方面来说, 单语家庭中成人的汉语教授强度以及儿童习得的汉 语经验在数量上要多于、内容上要丰富于儿童早期双语经验中的汉语经验或者英语经验。这 也许是早期双语者的词汇通达率低于单语者的原因之一。从另一方面来说, 双语儿童获得的 是双重经验, 当面对同一物质时, 儿童有可能仅对用英语命名感兴趣, 而对用汉语命名的同 一物质并不感兴趣。也就是说, 双语儿童实际上就是多了一次获得经验的机会, 认知更加灵 活, 能够从两种语言渠道中选择认识物质的方式。 
成人所了解的儿童语言习得质量远低于儿童真正的语言习得质量, 要了解语言习得的情况, 应该进行适当的诱发和干预。最常见的三种诱发方式为: 自由游戏、对话、故事。有研究表 明, 三种诱发方式所获得的语言习得结果不同, 就语言数量来看, 自由游戏多于对话、故事。

\section{【1】}

目前国内儿童汉语多在自由游戏中习得, 而第二语言大都在对话中习得, 比汉语习得更倾向 于正式环境。也就是说, 单语儿童其语言习得方式较为单一, 倾向于非正式; 双语儿童的语 言习得经常在非正式的汉语环境和较为正式的第二语言环境中根据需要来回切换。双语儿童 要运用两种语言分别适应于两种不同的环境, 这在无意识中锻炼了儿童的自我控制力、环境 适应力、和多重任务切换能力。

\subsection{2宏观系统}

西方国家以个人主义价值观为主, 中国是以集体主义价值观为主。我国在进行外语教学时, 会进行课程本土化处理。如教材、教案编写时采用不地道的语言、非真实的材料内容等。虽 然双语儿童与单语儿童所接受的是同一种价值观, 但是毕竟母语和外语的文化背景有差别。 双语儿童相对来说更能够接受不同的文化和种族, 有利于其独立认知结构的形成、认知分析 能力的提高和社会行为的发展, 如包容、平等等。

\section{2 输入途径}

语言感受是语言表达和运用的基础, 语言习得首先来源于语言感受。正常儿童的主要语言感 受器官为听觉器官和视觉器官。当代认知主义者将感觉系统纳入 “具身化”, 认为感官系统 与身体配置、跨感官的经验等在人类的认知和概念生产过程中担任着重要的基础角色。

儿童期是以具体形象思维为主, 视觉在语言习得中主要担任感知以及提供支架式理解帮助的 作用。就语言本身来说, 英文是拼音文字, 而汉语是图形文字, 二者学习具有不同的神经机 制。母语是图形文字的学习者在识别拼音文字的语言时, 更依赖于视觉字形信息, 但是在语 音意识上存在迁移, 在字形学习中不存在迁移, 这可能是因为所识别的不是图形文字; 母语 是拼音文字的学习者在识别拼音文字的语言时更依赖于语音信息。汉语是图形文字，学习第 二语言 (拼音文字英语) 时, 由于母语是图形文字所以依赖于视觉, 但是在视觉上不存在迁 移, 在听觉上存在迁移, 也就是说是以第二语言的特征来决定哪种感知觉存在迁移, 以第一 语言的特征决定习得过程依赖于哪种感知觉。比单语者更复杂的多模态感官系统联结会使双 语儿童的语言感受更多。视觉适应性研究发现, 双语儿童对熟悉图片的厌烦速度更快, 注视 时间更短, 对新奇事物的偏好率更高。【6】这说明双语儿童具有更高的认知潜力或者更大的 认知空间, 其注意力比单语者更容易集中到新事物上, 为双语者的创造力更强提供了依据。

\section{3 语言加工}

\section{3. 1 生理基础}

汉语学习和英语学习在儿童大脑中建构的图示不同, 双语儿童建构图示丰富于单语者。丰富 的图示可以为解决问题提供多方面的渠道, 促进问题的解决。人在习得某种知识技能时, 如 果脑区中已有图示的共同点较少, 同化已经满足不了加工需要, 此时便需要顺应, 即征用不 同的脑区、神经网络进行加工。但是儿童与青少年、成人的生理特点不同，儿童未完成大脑 侧化。新旧知识技能的经验联结能够在布洛卡区产生相似的神经表征, 而且大脑可重塑, 功 能区相互叠合。

有研究发现, 第二语言习得的越早, 母语神经网络参与的越多。【3】在大脑未完成侧化时, 通过具有不同特点的经验进行联结, 可以较为容易的激活并且征用不同的脑区进行迁移。认 知神经科学研究发现, 新知识技能的学习可以引起大脑功能重塑, 就大脑灰质密度而言, 五 岁以前学习第二语言大于十岁以后学习第二语言大于单语者。大脑灰质中含有神经细胞, 灰 质密度越大神经细胞越紧密, 联结强度越大。功能磁共振研究发现语言加工相关脑区的功能 活动模式与大脑结构能够预测第二语言的学习能力。这说明, 二者的变化会对学习能力产生 一定的影响。 


\subsection{2人格特质}

人格特质是指个体所具有的相对稳定的神经特质, 所谓的相对稳定是指人格特质即受先天遗 传因素的影响, 又能够后天塑造, 而后天塑造容易受到价值观的影响。中国较西方国家相对 感性、追求稳定、倾向于回避不确定性因素, 大部分中国人的人格更多的倾向于少阳、少阴、 尽责性人格, 西方人的人格则倾向于太阳性、外倾性、开放性。研究发现, 跨文化敏感度与 人格相关, 特别是与开放性、外倾性相关联。开放性、外倾性越明显, 其跨文化敏感度越低。 所以, 双语儿童在两种文化影响中, 其人格特质可能会得到塑造, 倾向于阴阳和平型人格, 具有较高的适应能力和多重任务管理能力, 其社会性行为也会得到发展。

1.3.3自我形象

人类两大高层次需要之一就是自我实现需要。它决定自我形象, 也就是说决定个人对自身的 评价一需要决定评价。习得第二语言有利于增强社交主动性。Guriora 提出习得第二语言能够 获得一种新的语言自我。【4】

社交主动性的增长能够减少环境型焦虑, 以及由环境型焦虑导致的性格焦虑, 它与新的语言 自我、自我形象的树立、自我实现需要的提高有关, 语言自我、自我形象、自我实现需要为 双语者的社交主动性提供了内在动机。社交主动性增长后便会有更广泛的交际活动, 更广泛 的交际活动需要复杂的移情, 移情会锻炼儿童的认知灵活性。

\subsection{4记忆}

U1man【5】等人认为, 陈述性记忆系统与程序性记忆系统在第二语言学习中扮演的角色与母 语学习中扮演的角色不完全相同。研究发现, 在词汇学习中, 双语均主要依赖于陈述性记忆 系统; 在语法学习中, 母语依赖于程序性记忆系统, 第二语言依赖于陈述性记忆系统更多。 这种差别与双语习得的人文环境有关: 母语语法是无意识习得, 第二语言的语法是有意识习 得。第二语言除非原生态双语家庭, 否则很难达到双母语水平。也就是说, 双语者的陈述性 记忆系统能够较单语者更多地应用, 这也许是双语者的形状、空间记忆、短期记忆均优于单 语者的原因之一。

\section{2 课程理念启发}

双语者与单语学习者习得过程中存在诸多的差异, 这些差异也让双语者在认知能力、执行能 力以及解决问题的能力等方面更具有优势, 这就要求我们在普通课程中需要更加的关注习得 过程以及在过程中无意识习得的能为儿童一生所用的能力。

首先, 单纯的角色扮演以及混杂的情感体验是有失偏颇的。课程应该注重设置具有较大差异 性的情景元素混合体, 如环境设置方面的差异性、生理和感官方面的差异性、心理方面的差 异点等。课程应该尽量在有限的时间、资源、空间内为每个儿童提供两种差异性较大的角色、 情景代入体验, 帮助帮助儿童提高环境适应能力、自我控制力、多重任务管理和切换能力。 同时, 在儿童记忆能力的培养方面, 课程不应该仅注重程序性记忆系统, 涉及陈述性记忆如 儿童的短时记忆能力以及对空间、形状、情景元素、语音等的记忆也应该得到发展, 记忆力 越早得到发展, 意识就能够越早代替记忆。

此外, 课程引导儿童树立的价值观应是将中西文化取精华去糟粕的半集体半个人主义, 此课 程理念可以渗透至 6 岁左右大班的小组课程和集体课程中。为解决纠纷冲突提供基础, 促进 社会性行为的发展 (包括包容、平等等) 以及阴阳和平型人格的形成。

再者, 儿童的个体需求决定了个体对自身的评价 (即: 自我形象), 课程应该重视不同个体的 需求种类、需求水平等, , 根据不同的个体需求创设多层次的课程, 引导儿童个体进行自我 形象的树立以及教师进行儿童形象的树立, 为儿童的社交行为提供内在动机。 


\section{References}

[1]. Xiaosong Ge, Wei Yang, Yu Tai. Analysis techniques for children's language samples [J]. Progress in psychological science, 2009, 17 (06): 1242-1249.

[2]. Fenghua Zhang, Xingming Jin, Xiaoming Shen, et al. Early intervention in language, speech and cognition of infants with hearing impairment [J]. Chinese Journal of medicine, 2006 (40): 2836-2840.

[3]. Li Yan. Metalinguistic awareness and personality traits [J]. Journal of Hunan Institute of socialism, 2015, 16 (06): 92-94.

[4]. Xueyun $\mathrm{Wu}$. Affective factors affecting second language acquisition [J]. Foreign language audio-visual instruction, 1997 (04): 21-23+33.

[5]. Ullman MT.A neurocognitive perspective on language: The declarative procedural model [J].Nature Reviews Neuroscience, 2001, Vol.2 (10), pp.717-726. 